\title{
Biomarcadores en vesículas extracelulares: Una alternativa para el diagnóstico del Cáncer Colorrectal
}

\author{
Biomarkers in extracellular vesicles: An alternative for the diagnosis of Colorectal \\ Cancer
}

\author{
Marco Antonio Hernández Bedolla ${ }^{a}$, Dulce Edith Alonzo Alonso ${ }^{b}$, Itzel Guadalupe Díaz \\ Salguero $^{c}$, Emmanuel Reyes Uribe ${ }^{d}$, Alma Barajas-Espinosa ${ }^{e}$, Fernando Ochoa-Cortés $^{f}$
}

\begin{abstract}
:
Colorectal cancer (CRC) is a disease characterized by the uncontrolled growth of cells in any of the four portions of the colon or in the rectum, it is a public health problem throughout the world. In Mexico, it is the third most prevalent cancer and ranks second place with regards to mortality. The main inherent risk factor for $\mathrm{CRC}$ is increasing age. Modifiable and non-modifiable risk factors are present in this pathology, leading to limited evidence of their presence. Among the diagnostic options that are currently available, options for stool tests and endoscopic and / or radiological visual examinations. Biomarkers with characteristics of specific biological, pathogenic, or pharmacological processes have been shown to be objective indicators and have been reported to improve the diagnosis, prognosis, and therapy of CRC. In this review, we will illustrate the main biomarkers identified in extracellular vesicles isolated from tissue affected by CRC.
\end{abstract}

Keywords:

Biomarkers, extracellular vesicles, colorectal cancer, diagnosis

Resumen:

El cáncer colorrectal (CCR), una enfermedad caracterizada por el crecimiento descontrolado de células en cualquiera de las cuatro porciones del colon o en el recto, es un problema de salud pública en todo el mundo. En México, este cáncer ocupa el tercer lugar con respecto a incidencia y el segundo lugar con respecto a defunciones. El principal factor de riesgo inherente para el CCR es la edad avanzada. Se presentan factores de riesgo modificables y no modificables en esta patología que lleva a tener evidencias limitadas de su presencia. Dentro de las opciones de diagnóstico con las que se cuentan en la actualidad encontramos opciones de pruebas en heces y exámenes visuales endoscópicos y/o radiológicos. Se ha demostrado que los biomarcadores con características de procesos biológicos, patogénicos o farmacológicos específicos fungen como indicadores objetivos y su detección oportuna mejora el diagnóstico, el pronóstico y la terapia del CCR. En esta revisión, ilustraremos los principales biomarcadores identificados en vesículas extracelulares aisladas de tejido afectado por CCR.

\section{Palabras Clave:}

Biomarcadores, vesículas extracelulares, cáncer colorrectal, diagnóstico

\section{Introducción}

La incidencia y la mortalidad por cáncer crece rápidamente en todo el mundo, en el 2020 se registraron poco más de 19 millones de nuevos casos y cerca de 10 millones de defunciones ${ }^{1}$. Las razones son complejas, pero pueden estar asociadas al envejecimiento, crecimiento de la población, así

\footnotetext{
a Autor de Correspondencia, Universidad Autónoma del Estado de Hidalgo, Email: marco_hernandez@uaeh.edu.mx

b Universidad Autónoma del Estado de Hidalgo, https://orcid.org/0000-0001-6909-201X, Email: al326873@uaeh.edu.mx

c Universidad Autónoma del Estado de Hidalgo, Email: wiri0319@gmail.com

d Universidad de Guadalajara, https://orcid.org/ 0000-0002-3602-9273, Email: emmanuel.reyes@academicos.udg.mx

e Universidad Autónoma del Estado de Hidalgo, https://orcid.org/0000-0002-3961-3280, Email: alma_barajas@uaeh.edu.mx

f Universidad Autónoma del Estado de Hidalgo, https://orcid.org/0000-0002-1559-8012, Email: fernando_ochoa@uaeh.edu.mx
} 
como los cambios en la prevalencia y distribución de los principales factores de riesgo de cáncer y al desarrollo socioeconómico.

El cáncer colorrectal (CCR) es un problema de salud pública en todo el mundo, es una enfermedad caracterizada por el crecimiento descontrolado de células en cualquiera de las cuatro porciones del colon o en el recto. Generalmente, antes de la aparición del cáncer se desarrollan formaciones o lesiones premalignas de tejido en la pared interna del colon o recto, llamadas pólipos; estas formaciones pueden aparecer en cualquier porción del intestino grueso y tardaran entre 10 y 15 años en crecer a un tamaño detectable. Con el tiempo, los pólipos pueden transformarse en cáncer (adenocarcinoma) lo que los hacen idóneos a prevención secundaria, es decir, a ser diagnosticado en etapas tempranas de la patología ${ }^{2,3}$.

El principal factor de riesgo inherente para el CCR es la edad avanzada, presentándose con mayor frecuencia en individuos mayores de 50 años ${ }^{4}$. Las estrategias de prevención primaria para reducir la incidencia de CCR incluyen implementación de políticas públicas asociadas a la promoción de cambios de conducta individual, favoreciendo modificaciones en la dieta, práctica regular de ejercicio, mantenimiento de un peso saludable y reducción en el consumo de tabaco y alcohol ${ }^{5}$. La prevención secundaria para CCR se realiza a través de pruebas de tamizaje, como son: la sangre oculta en heces, rectosigmoidoscopia o colonoscopia que detectan pólipos que pueden eliminarse antes de que desarrollen cáncer. El tamizaje para CCR es una estrategia efectiva para reducir su mortalidad en aproximadamente un promedio del $20 \%{ }^{6,7}$. Cuando el CCR se detecta en etapas tempranas la tasa global de supervivencia puede alcanzar el 90\%, mientras que, cuando se diagnostica en etapas avanzadas, las posibilidades de supervivencia son considerablemente menores ${ }^{8}$.

Aunque la evidencia acumulada de numerosos estudios ha demostrado estrategias de diagnóstico temprano, terapia y predicción del pronóstico en el CCR; actualmente sigue siendo un gran desafío proporcionar una guía clínica objetiva para los pacientes individuales ${ }^{9,10}$. Se ha demostrado que los biomarcadores con características de procesos biológicos, patogénicos o farmacológicos específicos son indicadores objetivos y su aplicación mejora el diagnóstico, el pronóstico y la terapia del $\mathrm{CCR}^{11,12,13}$. Durante las últimas décadas, la investigación en la temática del cáncer se ha centrado en el estudio de nuevos biomarcadores y sus funciones fisiológicas. Nuestro grupo de investigación busca determinar nuevos biomarcadores en pacientes con CCR, a partir del análisis de las vesículas extracelulares que son un grupo heterogéneo de vesículas limitadas por membrana cargadas con diversas proteínas, lípidos y ácidos nucleicos. De tal manera, el objetivo de esta revisión es dar a conocer los principales biomarcadores identificados de vesículas extracelulares aisladas del CCR.

\section{Cáncer Colorrectal}

\subsection{Anatomía del colon}

El colon o intestino grueso es el último segmento del tubo digestivo. Tiene una longitud aproximada de 1,5 metros y se extiende desde el final del intestino delgado hasta el ano. Su diámetro es mayor en su origen (colon ascendente, diámetro de $7 \mathrm{~cm}$ ) y disminuye en su trayectoria (colon transverso y descendente, diámetro de $4 \mathrm{~cm}$ ), solo para dilatarse otra vez al llegar al recto, originando la ampolla rectal. El colon derecho se inicia en el ciego, continúa con el colon ascendente, el ángulo hepático y termina en el colon transverso proximal ${ }^{14,15}$. El colon izquierdo comprende la porción distal del colon transverso, ángulo esplénico, colon descendente, sigmoides, rectosigmoides y canal anal. El colon transverso y el sigmoides están suspendidos en la cavidad peritoneal por tejido conectivo, los mesenterios, en tanto que el colon ascendente y el descendente están fijos al retroperitoneo ${ }^{14,15,16}$. Diversas funciones se le han atribuido, dependiendo la zona del colon que se refiera. La función principal del colon ascendente y transverso es la absorción de agua y electrolitos $\left(\mathrm{Na}^{+}, \mathrm{K}^{+}\right.$, etc.), mientras que en el colon descendente y recto se almacenan las materias fecales antes de su expulsión por el ano ${ }^{17}$. Tanto el colon como el recto están constituidos por varias capas de tejido, la más interna es la mucosa, seguida por la submucosa, y más externamente se sitúa la capa muscular (su contracción logra el avance del contenido del tubo digestivo) que a su vez está recubierta por la serosa (capa más externa). En la mucosa existen glándulas productoras de moco que protegen el interior del colon y del recto, en ellas es donde se producen cerca del $95 \%$ los tumores malignos ${ }^{14}$. Este proceso comienza con una cripta aberrante, que evoluciona hacia una lesión precursora neoplásica (un pólipo) y eventualmente progresa a CCR durante un período estimado de 10 a 15 años. Actualmente se asume que la célula de origen de la mayoría de los cánceres colorrectales es una célula madre o una célula parecida a ella ${ }^{18,19}$.

\subsection{Epidemiología}

Se estipula que el CCR aumente debido al crecimiento y envejecimiento de la población, así como a la adopción de comportamientos y estilos de vida que incrementan el riesgo $^{20}$. Tomando de referencia la base de datos GLOBOCAN 2020 de la Agencia Internacional de Investigación en Cáncer (IARC) de la Organización Mundial de la Salud (OMS), se registra una incidencia de 1.93 millones de nuevos casos de CCR a nivel mundial, colocándolo en tercer lugar solo por detrás del de mama y pulmón. Se estima que para el 2040 la incidencia de CCR incremente a aproximadamente 3.15 millones de nuevos casos. El CCR es el segundo cáncer con mayor mortalidad (935,173 decesos en el año 2000) y se prevé que para el 2040 está tasa aumente a cerca de 1.62 millones de muertes. En México este tipo de cáncer es el tercero con mayor incidencia con un estimado de 14,901 casos y una proyección en 20 años de 25,800 nuevos casos. En el caso de la letalidad a nivel nacional esta patología ocupa el segundo lugar donde es de destacar el alto índice de mortalidad, de alrededor del 50\%. Mientras canceres con mayor incidencia como el de mama y próstata tienen un 
índice de letalidad de alrededor del 25-30\%. Se estima se visualiza que dentro de dos décadas se dupliquen los decesos en el país a consecuencia de este cáncer (figura 1). Los datos referidos son inclusivos de ambos sexos y de todas las edades $^{[1]}$.

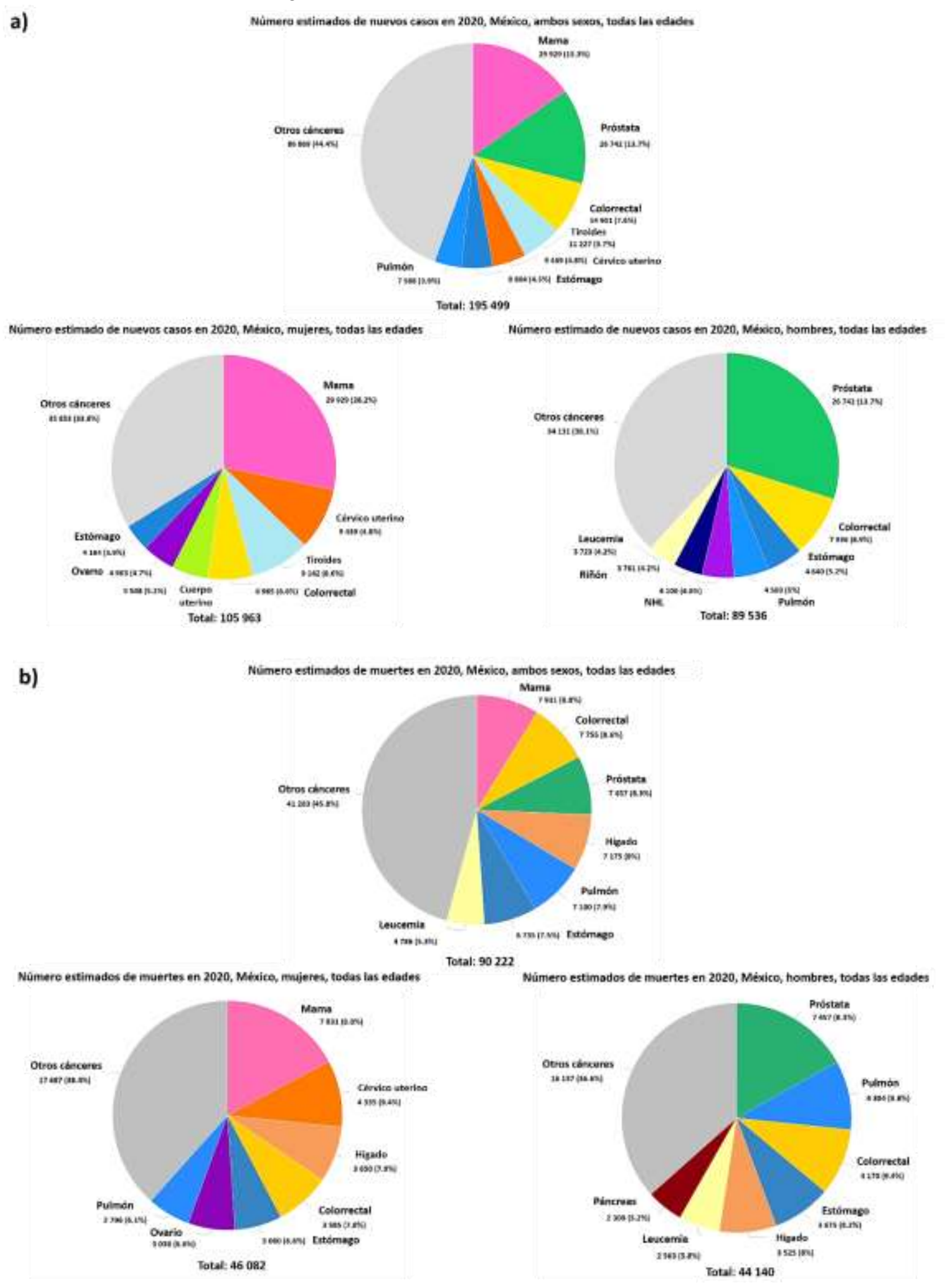

Figura 1. Incidencia y mortalidad por CCR en México. a) Se muestra la incidencia de nuevos casos de CCR en el país en 2020 colocándolo en tercer lugar con 14, 901 casos. Si hablamos por sexo, en mujeres se sitúa en cuarto lugar con 6,695 nuevos casos, por detrás del cáncer de mama, cervicouterino y tiroides. La incidencia en hombres lo posiciona en segundo lugar con 7,936 nuevos casos solo después del cáncer de próstata. b) Se muestra la mortalidad por CCR en el país en 2020 colocándolo en segundo lugar con 7, 751 casos. Si hablamos por sexo en mujeres se sitúa en cuarto lugar con 6, 585 muertes, por detrás del cáncer de mama, cervicouterino e hígado. La mortalidad en hombres 
lo posiciona en tercer lugar con 4, 170 defunciones solo después del cáncer de próstata y pulmón. Los datos y gráficos en ambos incisos son para todas las edades. Imágenes adaptadas y traducidas de la base de datos Globocan 2020; hhtp://gco.iarc.fr

\subsection{Signos y síntomas}

Generalmente, los síntomas aparecen en etapas avanzadas, por lo que es importante estar atento a ellos. Encontramos cambios en la defecación por varios días, donde se presenta diarrea intermitente, estreñimiento y heces más delgadas ${ }^{16}$. Uno de los síntomas más frecuentes del CCR es el sangrado en heces, puede tratarse de sangre roja, más habitual en tumores de recto, sigma y colon descendente, o de sangre negra, que se mezcla con las heces dando lugar a deposiciones de color negro también llamadas melenas ${ }^{14}$. Las melenas aparecen con más frecuencia cuando el tumor está situado en el colon ascendente. Otros signos que son considerados importantes en el CCR son los cólicos o dolor abdominal, la sensación persistente de defecar y pérdida inexplicable de peso. Estos síntomas pueden ser ocasionados por otras enfermedades comunes $\mathrm{y}$, por lo tanto, frecuentemente son ignorados $5,17,21$.

\subsection{Factores de riesgo}

En estudios epidemiológicos, el sexo masculino e incremento de la edad han mostrado consistentemente una fuerte asociación con la incidencia de enfermedades. Tanto los factores hereditarios como los ambientales intervienen en el desarrollo del CCR (figura 2). Entre los factores de riesgo que no son modificables, se encuentran: la formación de pólipos en el colon; enfermedad inflamatoria intestinal y los antecedentes familiares que, parecen tener un papel en el 10-20\% de todos los pacientes con $\mathrm{CCR}^{22}$, según el número y grado de los parientes afectados y la edad del diagnóstico del $\mathrm{CCR}^{23}$. Varios factores ambientales incorporados en el estilo de vida son, mayoritariamente, modificables. Dichos factores incluyen: el tabaquismo, ingesta excesiva de alcohol, aumento de peso corporal, ingesta de carne roja y procesada. Aunque la diabetes tipo 2 y el CCR comparten algunos de los mismos factores de riesgo (como la obesidad, inactividad física), las personas con diabetes tipo 2 mantienen su mayor riesgo después de corregir estos factores ${ }^{24,25,26,27,28}$. Los estudios sobre la microbiota colónica sugiere que la infección con especies bacterianas específicas, como Fusobacterium nucleatum y Bacteroides fragilis, podría aumentar el riesgo de desarrollar $\mathrm{CCR}^{29,30}$.

\section{Detección y diagnóstico}

No existe un consenso categórico sobre la técnica preferida de tamizaje para neoplasias y CCR porque ninguna de las pruebas utilizadas es completamente idónea para todos los grupos de riesgo; además no todos los países cumplen con los requerimientos, infraestructura, personal y protocolos para llevar a cabo un programa de tamizaje correcto, tal es el caso en México. La elección de la prueba depende en muchos casos de su eficiencia y desempeño, conjuntamente del nivel de recursos del lugar donde se establezca el programa ${ }^{31}$. La colonoscopia es el estándar de oro, sin embargo, la recomendación general para un programa de tamizaje sería someter a la población a un proceso rápido, usando una prueba de sangre oculta en heces preferentemente una Prueba Inmunoquímica Fecal (FIT por sus siglas en inglés) y seleccionar a los individuos que obtengan un resultado positivo para una colonoscopia. Independientemente de la técnica utilizada, un programa organizado de tamizaje debe asegurar la calidad de desempeño y garantizar su alcance a proporciones altas de la población ${ }^{32}$. Dentro de las opciones con las que se cuenta en la actualidad encontramos opciones de pruebas en heces y exámenes visuales endoscópicos y/o radiológicos.

\subsection{Pruebas en Heces}

Los tumores cancerosos y algunos pólipos grandes sangran intermitentemente en el tracto intestinal. Esta sangre, que puede no ser visible, es detectable dentro de las heces mediante pruebas especiales ${ }^{5}$.

\subsubsection{Prueba de Sangre oculta en Heces tipo Guayaco (gFOBT)}

Estas pruebas emplean una reacción química para detectar sangre en las heces. El sangrado por CCR puede ser esporádico o indetectable, por lo que los resultados de las pruebas precisas requieren pruebas anuales de tres muestras de deposiciones consecutivas. Se debe instruir a los pacientes para que eviten los fármacos antiinflamatorios no esteroides (AINES) y la carne roja durante los tres días previos a la prueba, ya que pueden dar lugar a resultados falsos positivos (gFOBT detecta sangre de cualquier fuente, incluida la carne en la dieta). También se deben evitar la vitamina $\mathrm{C}$ y grandes cantidades de jugos cítricos, ya que también pueden dar lugar a falsos positivos $^{5}$.

\subsubsection{Prueba Inmunoquímica Fecal (FIT)}

La prueba FIT (también denominado a veces FOBT inmunoquímico, o iFOBT) utiliza anticuerpos contra la hemoglobina para detectar sangre oculta en las heces. Las primeras versiones de esta prueba no fueron tan efectivas para detectar el cáncer como las versiones actuales, altamente sensibles, que han estado en el mercado por más de 10 años ${ }^{33,34}$. FIT es más conveniente que gFOBT porque no requiere restricciones dietéticas (solo detecta sangre humana) y generalmente requiere la recolección de menos muestras de heces ${ }^{35}$. El FIT también es específico para el sangrado que se produce a nivel colorrectal, por lo tanto, tiene menos resultados falsos positivos que el gFOBT entre poblaciones con una alta prevalencia de infección por Helicobacter pylori, que puede causar sangrado estomacal ${ }^{5}$. 


\section{Factores de riesgo para el desarrollo de CCR}

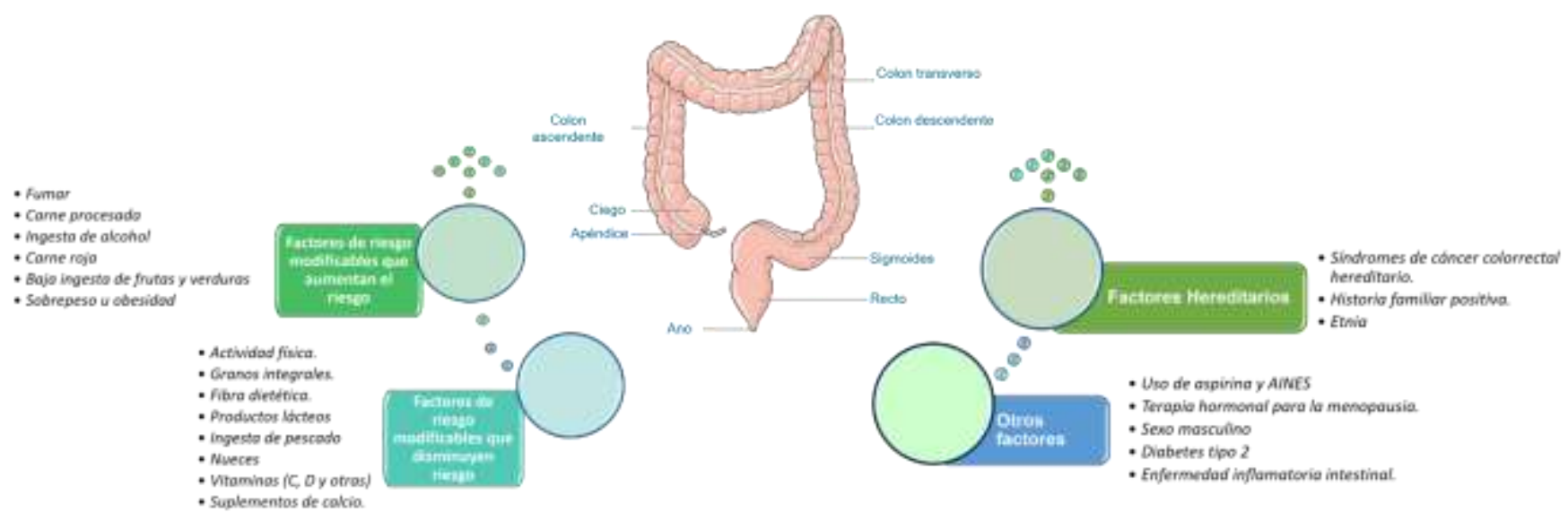

Figura 2. Factores de riesgo modificables y no modificables para el CCR. Se ilustra el grupo de factores hereditarios como aquellos de riesgo no modificables. Los factores de riego modificables existen dos tipos: los que aumentan el riesgo, tales como fumar, ingesta de alcohol, sobrepeso u obesidad, Diabetes tipo 2, terapia hormonal para la menstruación, entre otros; y los que disminuyen el riego encontramos la actividad física, fibra dietética, ingesta de pescado, vitaminas entre otros. La figura fue adaptada de Servier Medical Art, https://smart.servier.com/, y empleadas bajo Creative Commons Attribution 3.0.

\subsubsection{Prueba Fecal de ADN (FIT-DNA Cologuard $($ )}

Esta prueba se conoce como "multi-target" porque no solo detecta sangre en las heces, sino también ciertas mutaciones genéticas en el ADN de las células que se desprenden de las heces por adenomas grandes y CCR. Los pacientes con un resultado positivo de la prueba son referidos para una colonoscopia. Se ha demostrado que Cologuard ${ }^{\circledR}$ detecta el cáncer y las lesiones precancerosas con más frecuencia que la FIT, pero también da más resultados falsos positivos, repercutiendo en colonoscopias innecesarias ${ }^{36}$. Además, al tratarse de una prueba nueva, los beneficios y los riesgos asociados (si existieren), no se han establecido. Aunque está reconocida como una opción de evaluación aceptable y no todos los programas de seguridad en salud cubren el costo de esta prueba $^{37}$.

\subsection{Exámenes Visuales Endoscópicos 2.2.1. Colonoscopia}

Colonoscopia es un estudio eficiente de tamizaje para CCR siempre y cuando se realice bajo los criterios de calidad internacionales, esto implica cumplir con la normatividad vigente local y sólo se le considerará una colonoscopia completa (CC) hasta que se haya canulado el ciego con un tiempo de retirada mínimo de 7 minutos. Una CC permite la visualización directa de la mucosa colónica, biopsia de lesiones y extirpación de pólipos en todo el colon. La sensibilidad y especificidad para CCR y adenomas avanzados (AA) son muy altas. La CC es la prueba de confirmación utilizada en caso de una prueba positiva para todas las estrategias de detección anteriores. La eficacia de la CC muestra un impacto relevante en la incidencia (reducido en un 66-90\% en comparación con la población general) y la mortalidad $(31-65 \%)^{38}$. La colonoscopia como prueba de detección de CCR no está exenta de limitaciones. Se ha informado una alta variabilidad interoperacional en la tasa de detección de adenomas y esta característica se ha asociado con el riesgo posterior de $\mathrm{CCR}^{39}$. Por lo tanto, son necesarios programas de capacitación adecuados para los endoscopistas, así como un control continuo de la calidad. Los efectos colaterales de la CC son raros, pero más frecuentes que con una sigmoidoscopia flexible (SF). Muchas características propias del procedimiento afectan negativamente la aceptabilidad de la CC como prueba de detección de primera línea: es un examen invasivo, que requiere una limpieza intestinal exhaustiva, consume mucho tiempo, costosa y dolorosa ${ }^{38}$.

\subsubsection{Sigmoidoscopia Flexible (SF)}

Ensayos clínicos controlados aleatorizados han demostrado que el empleo de la SF reduce la mortalidad por CCR en un $22-31 \%$, además de disminuir la incidencia en un $18-23 \%$ a través de la inspección visual de la mucosa colónica, la toma de biopsias y la extracción de pólipos en el tracto distal del colon. Por obviedad el impacto del tamizaje con SF en las tasas de incidencia y letalidad de CCR se limita al colon distal. Una estrategia combinada que use SF y gFOBT / FIT no parece resolver el problema $^{38}$. En general, el SF es una prueba segura con reportes de tasas de complicaciones muy bajas y no requiere sedación del paciente.

\subsubsection{Sigmoidoscopia Rígida (SR)}


La SR ha estado en uso para la detección de CCR del área rectosigmoidea desde finales de 1930. El sigmoidoscopio rígido estándar tiene una profundidad de inserción potencial de $25 \mathrm{~cm}$, aunque la profundidad de inserción más común ha sido un promedio de $17-20 \mathrm{~cm}$ en la mayoría de las series ${ }^{40}$. Sí comparamos la SR con la SF es evidente el mayor alcance y tolerabilidad del segundo, lo cual promueve su uso con mayor frecuencia, además de que hoy en día, prácticamente todos los equipos de SF ofrecen la oportunidad de documentar digitalmente los procedimientos. Desde nuestra perspectiva, la SR debe de seguir considerándose como parte del armamentario diagnóstico, en especial con lesiones que ocupen el tercio medio y distal del recto.

\subsection{Exámenes visuales radiológicos \\ 2.3.1. Colonografía por tomografía computarizada TC o TAC (CTC) o Colonoscopia virtual}

La CTC es una opción radiográfica mínimamente invasiva para la detección del CCR. Utiliza la tomografía computada para generar imágenes bidimensionales (2D) que permiten una reconstrucción tridimensional (3D) adicional con la asistencia de la tecnología de un software. En conjunto, la evaluación de imágenes $2 \mathrm{D}$ y $3 \mathrm{D}$ permite una detección precisa de neoplasias ${ }^{4}$. La CTC es menos invasiva que la colonoscopia o la sigmoidoscopia, no requiere tiempo de recuperación y, en general, demora aproximadamente de 10 a 15 minutos en completarse. Los pacientes con pólipos de más de 5 milímetros u otros resultados anormales se envían para colonoscopia, óptimamente el mismo día para evitar la necesidad de una segunda preparación intestinal. Los estudios han demostrado que el beneficio de la CTC es similar a la colonoscopia para la detección de cáncer invasivo y pólipos de aproximadamente 1 centímetro o más, pero tiene una sensibilidad menor para pólipos más pequeños ${ }^{5}$.

\subsubsection{Enema de bario o colon por enema}

En esta prueba, se introduce sulfato de bario en un colon limpio a través del recto para llenar y abrir parcialmente el colon. Posteriormente, se introduce aire para expandir aún más el colon y se toman radiografías. Este método es menos sensible que la colonoscopia para visualizar pólipos pequeños o CCR. Si se observa un pólipo u otra anomalía, el paciente debe ser referido para una colonoscopia. El uso de este procedimiento se ha vuelto muy poco frecuente debido a la mayor disponibilidad de la colonoscopia ${ }^{5}$.

\section{Biomarcadores moleculares}

Un biomarcador se define como una molécula biológica capaz de fungir como indicador medible, extraíble de fluidos corporales o tejidos, mediante los cuales se puede identificar un proceso patológico o fisiológico particular, o de una afección o enfermedad ${ }^{41,42}$. Los biomarcadores son herramientas valiosas para la detección, el diagnóstico, el pronóstico del paciente y la elección del tratamiento del cáncer y otras enfermedades. También se pueden utilizar para localizar el tumor, detectar el avance del cáncer y determinar la mejor opción terapeutica ${ }^{43}$. Las alteraciones celulares pueden deberse a varios factores, que incluyen mutaciones de la línea germinal o somáticas, cambios transcripcionales y modificaciones postraduccionales.

Existe una enorme variedad de biomarcadores que pueden incluir proteínas (por ejemplo, una enzima o receptor), ácidos nucleicos (por ejemplo, un microARN u otro ARN no codificante), anticuerpos y péptidos, entre otras categorías. Un biomarcador también puede ser una colección de alteraciones, como la expresión génica, las firmas proteómicas y metabolómicas ${ }^{44}$. Los biomarcadores pueden detectarse en la circulación (sangre total, suero o plasma), secreciones o excreciones (heces, orina, esputo o secreción del pezón) y, por lo tanto, se pueden evaluar fácilmente de forma no invasiva $\mathrm{y}$ en serie, o pueden derivarse de tejidos, y requieren biopsia o imágenes especiales para su evaluación ${ }^{45}$.

Los biomarcadores genéticos pueden heredarse y detectarse como variaciones de secuencia en el ADN de la línea germinal aislado de sangre total, esputo o células bucales, o pueden ser somáticos e identificarse como mutaciones en el ADN derivado de tejido tumoral ${ }^{45}$. Existen varios marcadores moleculares que participan directamente en los mecanismos de proliferación de células neoplásicas y la secreción de glicoproteínas anormales contenidas en el moco producido en el desarrollo del $\mathrm{CCR}^{46,47}$.

\section{Vesículas extracelulares}

Las vesículas extracelulares (EV) son una familia heterogénea de vesículas limitadas por membrana que tienen un origen diverso. En 1983 Pan y Johnstone ${ }^{48}$ fueron de los primeros en describir las EV. Inicialmente, se demostró que la liberación de EV era parte de un mecanismo de eliminación para descartar materiales no deseados de las células. Investigaciones posteriores han demostrado que la liberación de EV también es un mediador importante de la comunicación intercelular que existe de manera fisiológica y también patológica ${ }^{49,50,51}$.

Las EV se clasifican actualmente de acuerdo a su biogénesis, tamaño y densidad. En este sentido, las microvesículas (MV) se generan por protrusiones de la membrana plasmática que son liberadas al espacio extracelular y tienen en promedio un diámetro entre $100 \mathrm{y}$ $1000 \mathrm{~nm}^{52}$. Otro proceso de liberación involucra la gemación hacia adentro de la membrana endosomal, lo que resulta en la formación de cuerpos multivesiculares (MVB), con exosomas liberados por fusión de la membrana externa de MVB a la membrana plasmática ${ }^{53,54}$. Las vesículas también pueden liberarse de estructuras nanotubulares que se extienden desde la membrana plasmática $^{55}$.

Los exosomas se generan a partir de endosomas que posteriormente forman cuerpos multivesiculares con un 
alto contenido de vesículas intraluminales (ILV). Cuando las ILV se liberan al espacio extracelular reciben el nombre de exosomas y tienen un tamaño entre 20 y $120 \mathrm{~nm}$. Los cuerpos apoptóticos se generan a partir de células en proceso de muerte celular programada y tienen un tamaño entre 1000 y $5000 \mathrm{~nm}^{56,57,58}$.

Las diferencias en cuanto a su biogénesis permiten que las EV contengan diversas moléculas asociadas a su membrana o en su interior (proteínas, mRNAs, lncRNAs, miRNAs gDNA, lípidos, etc.), muchas de estas moléculas son funcionales y pueden transferirse a otras células de manera paracrina o endocrina, inclusive de manera autocrina, y desencadenar una respuesta biológica.

Se han llevado a cabo diversas investigaciones para caracterizar y determinar el contenido de las EV, lo que ha resultado en la creación de bases de datos de acceso público con una gran cantidad de información al respecto, que incluyen el contenido de proteínas, ácidos nucleicos y lípidos junto con los procedimientos de aislamiento y purificación. Entre la cuales podemos mencionar ExoCarta (http://www.exocarta.org), (http://www.microvesicles.org/) y $\quad$ EVpedia (http://evpedia.info) ${ }^{59}$.

\section{Biomarcadores en CCR}

La búsqueda de marcadores moleculares en CCR ha sido de gran interés en los últimos años. Una extensa investigación ha revelado que el CCR se desarrolla a través de tres vías principales: (1) anomalías cromosómicas que conducen a mutaciones de oncogenes y genes supresores de tumores (vía clásica), caracterizadas por la progresión del adenoma-carcinoma; (2) la vía de inestabilidad de microsatélites que resulta de defectos en el sistema de reparación del ADN; y (3) modificaciones postraduccionales y epigenéticas.

Cientos de moléculas involucradas en la vía de inestabilidad cromosómica se han asociado con el pronóstico; sin embargo, solo un marcador único, la vía del receptor del factor de crecimiento epidérmico (EGFR), ha demostrado con éxito su utilidad clínica hasta la fecha ${ }^{60}$, en gran parte debido a la complejidad y redundancia de las vías celulares, así como la falta de terapias que puedan apuntar a los diferentes biomarcadores. Si bien la investigación clínica continua hacía nuevas modalidades de tratamiento, sigue siendo imperativo encontrar biomarcadores moleculares que permitan detectar de manera no invasiva y en fases tempranas el CCR, así mismo, biomarcadores que den la opción de generar terapias más eficaces.

La vía del EGFR está implicada en la vía de inestabilidad cromosómica, y es un blanco terapéutico principal en el CCR localmente avanzado. Sin embargo, este tratamiento sólo es útil para pacientes con el homólogo del oncogén viral del sarcoma 2 de rata de Kirsten v-Ki-ras2 (KRAS) de tipo salvaje (wt-KRAS) ${ }^{61,62}$. La activación anormal de las vías de señalización del EGFR en el CCR se asocia principalmente con tres mutaciones en las vías de la proteína quinasa activada por mitógenos (MAPK) y la fosfatidilinositol-3-quinasa (PI3K): KRAS, el homólogo del oncogén viral RAS (v-ras) de neuroblastoma (NRAS) y el homólogo B1 del oncogén viral del sarcoma murino vraf (BRAF); estas tres mutaciones ocurren en más de la mitad de todos los casos de $\mathrm{CCR}^{63}$.

La mutación de algunos de los componentes de la vía EGFR, específicamente BRAF V600E ${ }^{64}$, KRAS (exón 2, $3,4)$ y la mutación NRAS (exón 2, 3, 4) hacen que las células malignas se vuelvan resistentes a la terapia antiEGFR; por lo tanto, los pacientes no deben ser tratados ni con cetuximab ni con panitumumab. Como resultado, todos los pacientes con CCR metastásico deben someterse a una investigación del estado de la mutación de KRAS / NRAS y BRAF antes del inicio del tratamiento.

Actualmente, se realiza un esfuerzo intenso en la identificación de biomarcadores nuevos para la detección de CCR en sangre, heces y otros fluidos biológicos. Hay tres tipos principales de marcadores: de pronóstico, predicción y diagnóstico, sensibles y específicos que se están desarrollando ${ }^{46,47}$ :

Diagnóstico: estos marcadores se utilizan para la estratificación del riesgo y la detección temprana de pólipos colorrectales.

Pronóstico: estos marcadores dan una indicación de la probable progresión de la enfermedad.

Predictivo: este tipo de marcadores moleculares se utilizan para predecir las medidas de tratamiento que se deben tomar en un paciente.

Se enlistan los biomarcadores más estudiados a la fecha y clasificados según su tipo (Figura 3):

\section{Biomarcadores en VE de CCR}

En algunos tipos de cáncer se ha observado que las células tumorales liberan una mayor cantidad de EV comparadas con células normales. Además, su contenido molecular es diferente y hace posible que existan moléculas que posean las características de un biomarcador ${ }^{65}$.

\section{1. miRNAs}

Los miRNA son RNA pequeños no codificantes de aproximadamente 18 a 25 nucleótidos (nt) de longitud. Se han reportado algunos miRNAs expresados en VE sanguíneas de pacientes con CCR que pueden ser biomarcadores potenciales para el diagnóstico clínico. Se han encontrado que siete miRNA (miR-23a, miR-1246, let7a, miR-1229, miR-150, miR-223 y miR-21) están altamente expresados en las VE séricas de pacientes con CCR, pero disminuyen significativamente después de la resección quirúrgica ${ }^{66}$. Los miR-23a, miR-1246 y miR-21 han mostrado una mejor precisión diagnóstica para el CCR que los demás. Las curvas de característica operativa del receptor (ROC) son herramientas poderosas para evaluar el rendimiento de los biomarcadores de diagnóstico, y el valor del área bajo la curva (AUC) representa su precisión general ${ }^{67}$. Cuanto más cercano esté el valor de AUC a 1,0, mayor será la sensibilidad y especificidad ${ }^{68}$. Las curvas ROC muestran que el AUC de miR-23a, miR-1246 y miR21 son $0,953,0,948$ y 0,798 , respectivamente. 


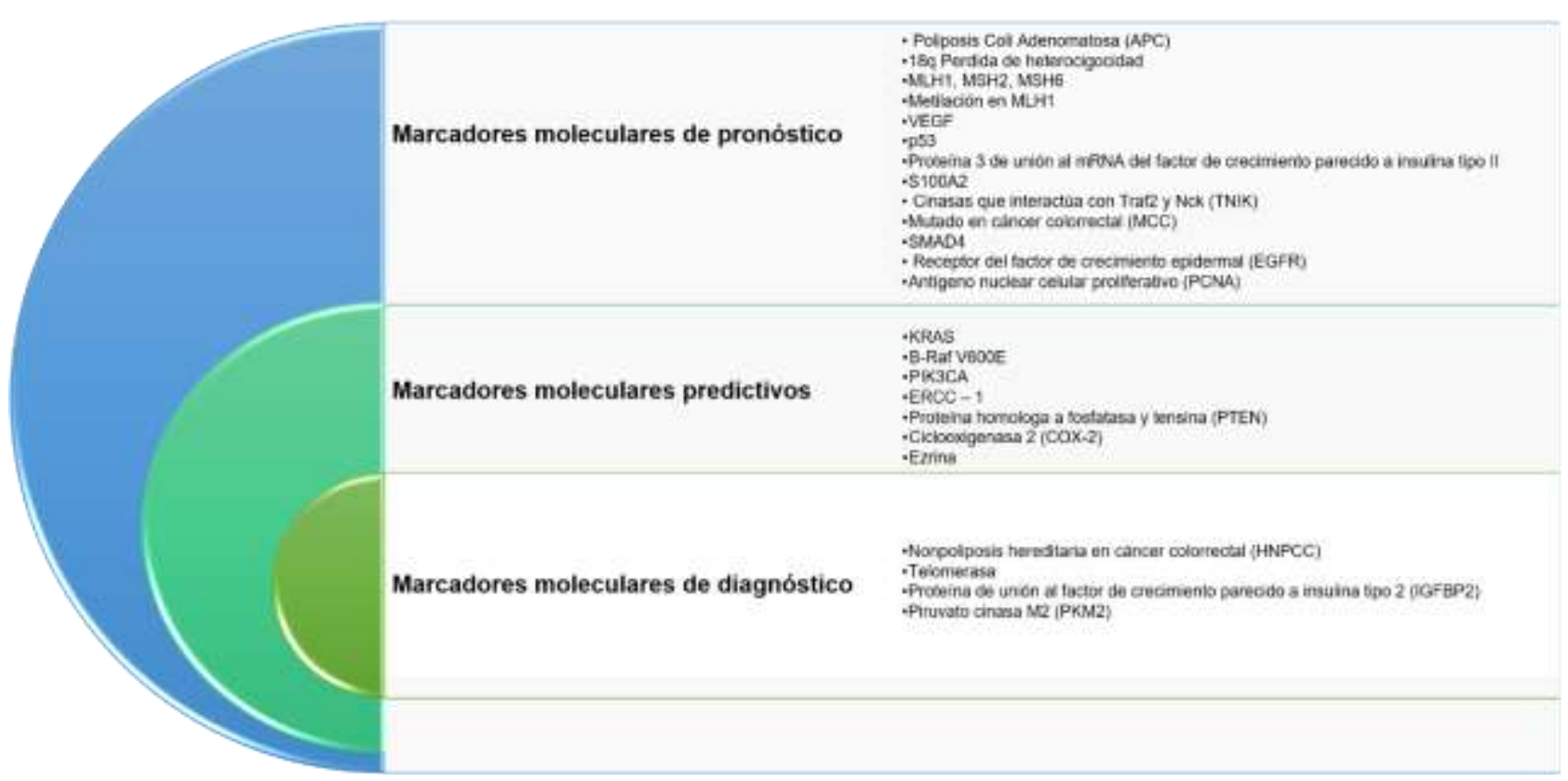

Figura 3. Biomarcadores moleculares para CCR. Se representa los biomarcadores moleculares más estudiados a la fecha y clasificados de acuerdo con sus características de estudio, enfoque y uso para el CCR.

En otro estudio identificaron 11 miRNA que se expresaron de manera diferencial en las VE séricas de pacientes con CCR. Descubrieron que la alta expresión de miR-23a y miR-301a en VE en pacientes con CCR. Los valores de AUC para miR-23a y miR-301a fueron 0,900 y 0,840 , respectivamente ${ }^{69}$. En otro trabajo, los niveles de miR-486$5 \mathrm{p}$ aumentaron notablemente en VE plasmáticas de pacientes con $\mathrm{CCR}^{70}$. El AUC de miR-486-5p para discriminar a los pacientes con CCR de los normales controles (NC) fue de 0,713. De manera similar, también se encontró que miR-6803-5p estaba claramente elevado en VE séricas, considerándose un índice de diagnóstico potencial en pacientes con $\mathrm{CCR}^{71}$. Otra investigación examinó sistemáticamente los RNA en VE plasmáticas de pacientes con CCR se identificaron biomarcadores específicos mediante secuenciación de RNA. Tres miRNA (let-7b-3p + miR-139-3p + miR-145-3p) se enriquecieron significativamente en VE plasmáticas ${ }^{72}$.

Otro estudio encontró 8 miRNAs en VE aisladas de células epiteliales por ensayos de inmunoafinidad hacía el marcador EpCAM. Los pacientes con CCR mostraron un aumento en los niveles de expresión hacía estos miRNAs, mientras que los controles sanos mostraron una menor expresión. Especulan que esos miRNAs son de origen tumoral y pueden tener potencial como biomarcadores no invasivos. La comparación de mucosa normal y tejido tumoral indica que 5 de los 8 miRNAs están regulados negativamente (miR-16-5p, miR-23b-3p, miR-27b-3p, miR-30b-5p y miR-30c-5p) y 3 regulados positivamente en tumores (miR23a-3p, miR-27a-3p y miR-222-3p). Esta desregulación en su expresión puede ser consistente con los niveles aumentados o disminuidos en la sangre de pacientes con CCR ${ }^{73}$.

Los microARN circulantes contenidos en VE (VE-
miRNA) han cobrado importancia recientemente como nuevos marcadores de diagnóstico del cáncer. Diferentes miRNAs se han encontrado altamente o poco expresados en las VE aisladas del suero o plasma de pacientes con CCR. Entre los que se encuentran altamente expresados están miR-1246, miR-21, miR-23a, miR-92a, miR-486-5P, miR-17, miR-19A y miR-125A-3P. En el caso de los miRNAs poco expresados se han reportado a miR-548C$5 \mathrm{P}$ y miR-125A-5P. En el caso de los VE-miRNAs obtenidos a partir líneas celulares de CCR se tienen los siguientes miRNA sobre regulados: miR-1246, miR-21, miR23a, miR-200c, miR-203a, miR-145, miR-17, miR19 a y $\mathrm{miR}-7641^{74}$.

\subsection{IncRNAs}

Los lncRNA, son RNA no codificante de más de 200 nt de longitud, regulan una plétora de procesos biológicos fundamentales (es decir, ciclo celular, remodelación de la cromatina, modificaciones de histonas, empalme y apoptosis) en diferentes niveles (transcripcional, postranscripcional, traslacional, postraduccional y epigenético $)^{75}$.

Se han encontrado expresados en las VE sanguíneas de pacientes con CCR. Se ha informado de que la sobreexpresión del lncRNA de la neoplasia colorrectal diferencialmente expresada-h (CRNDE-h) en las VE séricas de pacientes con CCR puede servir como un biomarcador potencial ${ }^{76}$. Otro estudio confirmó que el lncRNA del receptor de resistencia a los anti-estrógenos tipo 4 en del cáncer de mama (BCAR4), el mRNA de la proteína 5-4 asociada a la queratina (KRTAP5-4) y el mRNA de la familia A3 de antígenos de melanoma (MAGEA3) aumentaron en las VE séricas de los pacientes 
con $\mathrm{CCR}^{77}$. Un estudio reciente demostró que la disminución en la expresión del lncRNA 1 asociado a cáncer urotelial (UCA1) y sobre regulación del circRNA de la proteína quinasa 3 que interactúa con el homodominio (HIPK3) en las VE séricas de pacientes con CCR podrían ser candidatos para el diagnóstico no invasivo ${ }^{78}$

\subsection{Proteínas}

Muchos estudios han revelado la expresión diferencial de proteínas en VE sanguíneas de pacientes con CCR y controles sanos. En un estudio, se encontró que 36 proteínas estaban reguladas positivamente y 22 reguladas negativamente en las VE derivadas del suero de pacientes con CCR. Las proteínas sobre reguladas participan en procesos que modulan el microambiente tumoral y la metástasis, mientras que las proteínas reguladas negativamente participan principalmente en el crecimiento y la supervivencia de las células tumorales ${ }^{79}$.

Otro trabajo mostró que la proteína de choque térmico 60 (Hsp60) en VE plasmáticas de pacientes, es un candidato prometedor para el diagnóstico de CCR. Hsp60, una chaperona involucrada en la tumorogénesis, se expresa en gran medida en VE plasmáticas de estos pacientes, pero obviamente disminuye después de la cirugía ${ }^{80}$. Recientemente, se sugirió que VE plasmáticas positivas para glipicano-1 (GPC1) de pacientes con CCR son un indicador específico para el diagnóstico. El porcentaje de VE-GPC1 (+) y la expresión de la proteína GPC1 en estos $\mathrm{VE}$ aumentaron notablemente, pero ambos se normalizaron después de la cirugía ${ }^{81}$. Una investigación descubrió niveles significativamente elevados de VE positivas para la proteína de transmembrana CD147 en el plasma de pacientes con CCR. La curva ROC indicó que la concentración de exosomas CD147 (+) en plasma tenía una excelente capacidad de diagnóstico con un AUC de $0,932^{65}$. Además, un estudio propuso que la sobre expresión de la proteína copina III (CPNE3) en VE plasmáticas de los pacientes podría ser un biomarcador eficaz para el diagnóstico de $\mathrm{CCR}^{82}$.

Finalmente, en otro estudio el perfil de expresión del proteoma VE sérico en los pacientes con CCR de la porción ascendente es diferente al de los pacientes con CCR de la porción descendente. Las VE derivadas del suero de CCR de la porción ascendente promueven metástasis más significativas que los de la porción descendente. Esta diferencia puede atribuirse a la regulación positiva VE que contienen proteínas relacionadas con la matriz extracelular, especialmente proteoglicanos como la proteína ácida secretada y rica en cisteína (SPARC); y glicoproteínas como la alfa-2-glucoproteína 1 rica en leucina (LRG1). Las VE ricas en SPARC y LRG1 son biomarcadores de pronóstico y diagnóstico potencialmente no invasivo en cáncer de colon ${ }^{83}$.

\section{Conclusión}

El presente trabajo da una perspectiva sobre la importancia que tiene el CCR como un problema grave de salud pública a nivel mundial, se sitúa entre las 3 principales patologías oncológicas tanto en incidencia como en mortalidad. Una de las desventajas que tiene este tipo de cáncer es que sus síntomas aparecen a edades avanzadas y suelen confundirse con otras enfermedades. Su diagnóstico se basa en la colonoscopia y pruebas bioquímicas y moleculares en heces. Con lo anteriormente referido, muchos grupos científicos están buscando nuevas alternativas y herramientas de diagnóstico temprano para el CCR, esto a partir de diferentes muestras biológicas como sangre, orina, heces, esputo y secreciones del pezón. Actualmente hay otra vertiente de estudio y es donde se encuentra nuestro grupo de trabajo, buscamos estudiar elementos moleculares más selectivos como las vesículas extracelulares para dar a conocer nuevos biomarcadores, esto lleva a emplear técnicas de proteómica y genómica para dilucidar el perfil de las nuevas moléculas de estudio y sean candidatas a blancos de diagnóstico y tratamiento.

\section{Financiamiento:}

Este trabajo fue realizado con fondos otorgado a MAH-B por la Secretaria de Educación Pública, a través del Programa para el Desarrollo Profesional Docente, para el Tipo Superior, proyecto PRODEP-2019-0067. IGD-Z recibió una beca de estudiante bajo el mismo proyecto.

\section{Agradecimientos:}

Para el manejo de la literatura se utilizó la versión gratuita de Mendeley Cites Add-In Microsoft Word y Mendeley Reference Manager v1.19.4, Copyright (C) 2020 Mendeley Ltd. (Elsevier Inc., New York, NY, USA).

\section{Referencias:}

[1] World Health Organization, "Cancer Today," Int. Agency Res., vol. 418, pp. 1-2, 2020, Accessed: Jan. 13, 2021. [Online]. Available: https://gco.iarc.fr/today/.

[2] M. Vogelstein, B., Fearon, E. R., Hamilton, S. R., Kern, S. E., Preisinger, A. C., Leppert, "Genetic Alterations during Colorectal-Tumor Development," N. Engl. J. Med., vol. 319, no. $9, \quad$ pp. 525-532, 1988, doi: 10.1056/NEJM198809013190901.

[3] F. López-Köstner et al., "Programa de detección de neoplasias colorrectales en población mayor de 50 años," Rev. Med. Chil., vol. 140 , no. 3, pp. 281-286, 2012, doi: 10.4067/S003498872012000300001.

[4] C. B. Steele, S. R., Hull, T. L., Read, Th. E., Saclarides, T. J., Senagore, A. J., Whitlow, "The ASCRS Textbook of Colon and Rectal Surgery," ASCRS Textb. Colon Rectal Surg., p. 1292, 2016, doi: 10.1007/978-1-4419-1584-9.

[5] American Cancer Society, "Colorectal Cancer Facts \& Figures 2020-2022," Www.Cancer.Org, no. page 32, p. 48, 2020, [Online]. Available: https://www.cancer.org/content/dam/cancer$\mathrm{org} /$ research/cancer-facts-and-statistics/colorectal-cancerfacts-and-figures/colorectal-cancer-facts-and-figures-20202022.pdf.

[6] S. J. Winawer, "Natural history of colorectal cancer," Am. J. Med., vol. 106, no. 1 A, 1999, doi: 10.1016/s00029343(98)00338-6.

[7] D. K. Rex, D. A. Johnson, J. C. Anderson, P. S. Schoenfeld, C. A. Burke, and J. M. Inadomi, "American college of gastroenterology guidelines for colorectal cancer screening 2008," Am. J. Gastroenterol., vol. 104, no. 3, pp. 739-750, 
2009, doi: 10.1038/ajg.2009.104.

[8] J. B. O'Connell, M. A. Maggard, and C. Y. Ko, "Colon cancer survival rates with the new American Joint Committee on Cancer sixth edition staging," J. Natl. Cancer Inst., vol. 96, no. 19, pp. 1420-1425, 2004, doi: 10.1093/jnci/djh275.

[9] L. A. Torre, R. L. Siegel, E. M. Ward, and A. Jemal, "Global cancer incidence and mortality rates and trends - An update," Cancer Epidemiol. Biomarkers Prev., vol. 25, no. 1, pp. 16-27, 2016, doi: 10.1158/1055-9965.EPI-15-0578.

[10] C. L. Sawyers, "The cancer biomarker problem," Nature, vol. 452, no. 7187, pp. 548-552, 2008, doi: 10.1038/nature06913.

[11] J. K. Aronson, "Biomarkers and surrogate endpoints," $\mathrm{Br}$. J. Clin. Pharmacol., vol. 59, no. 5, pp. 491-494, 2005, doi: 10.1111/j.1365-2125.2005.02435.x.

[12] K. F. Newton, W. Newman, and J. Hill, "Review of biomarkers in colorectal cancer," Color. Dis., vol. 14, no. 1, pp. 3-17, 2012, doi: 10.1111/j.1463-1318.2010.02439.x.

[13] M. Verma and V. Kumar, "Epigenetic Biomarkers in Colorectal Cancer," Mol. Diagnosis Ther., vol. 21, no. 2, pp. 153-165, 2017, doi: 10.1007/s40291-016-0244-x.

[14] et. al. Eguino Villegas, Ana. Fernández Crespo, Ana Isabel, "Cáncer colorrectal Guía práctica," Aecc, vol. 12, pp. 9-18, 2002.

[15] D. Gallot, "Anatomía quirúrgica del colon,” EMC - Técnicas Quirúrgicas - Apar. Dig., vol. 22, no. 3, pp. 1-9, 2006, doi: 10.1016/s1282-9129(06)47081-1.

[16] SEOM: Sociedad Española de Oncología Médica (c) 2019, “Cáncer de colon y recto," Jan. 14, 2020. https://seom.org/infosobre-el-cancer/colon-recto (accessed Jan. 14, 2021).

[17] Asociación Española contra el Cáncer, "Cáncer de colon| AECC," 2018. https://www.aecc.es/es/todo-sobre-cancer/tiposcancer/cancer-colon (accessed Jan. 14, 2021).

[18] D. Nassar and C. Blanpain, "Cancer Stem Cells: Basic Concepts and Therapeutic Implications," Annu. Rev. Pathol. Mech. Dis., vol. 11 , no. 1, pp. 47-76, May 2016, doi: 10.1146/annurevpathol-012615-044438

[19] J. P. Medema, "Cancer stem cells: the challenges ahead.," Nat Cell Biol., vol. 15, no. 4, pp. 338-344, 2013, doi: $10.1038 /$ ncb2717.

[20] F. Bray, J. Ferlay, I. Soerjomataram, R. L. Siegel, L. A. Torre, and A. Jemal, "Global cancer statistics 2018: GLOBOCAN estimates of incidence and mortality worldwide for 36 cancers in 185 countries," CA. Cancer J. Clin., vol. 68, no. 6, pp. 394424, 2018, doi: 10.3322/caac.21492.

[21] I. American Cancer Society, "Acerca del cáncer colorrectal," pp. 1-15, 2018.

[22] N. B. Henrikson et al., "Family history and the natural history of colorectal cancer: Systematic review," Genet. Med., vol. 17, no. 9, pp. 702-712, 2015, doi: 10.1038/gim.2014.188.

[23] R. E. Schoen et al., "Incidence and Mortality of Colorectal Cancer in Individuals with a Family History of Colorectal Cancer," Gastroenterology, vol. 149, no. 6, pp. 1438-1445.e1, 2015, doi: 10.1053/j.gastro.2015.07.055.

[24] E. Botteri, S. Iodice, V. Bagnardi, S. Raimondi, A. B. Lowenfels, and P. Maisonneuve, "Smoking and Colorectal Cancer," Jama, vol. 300, no. 23, p. 2765, 2008, doi: 10.1001/jama.2008.839.

[25] S. Cai, Y. Li, Y. Ding, K. Chen, and M. Jin, "Alcohol drinking and the risk of colorectal cancer death: A meta-analysis," Eur. J. Cancer Prev., vol. 23, no. 6, pp. 532-539, 2014, doi: 10.1097/CEJ.0000000000000076.

[26] M. Kyrgiou et al., "Adiposity and cancer at major anatomical sites: Umbrella review of the literature," BMJ, vol. 356, pp. 110, 2017, doi: 10.1136/bmj.j477.

[27] D. S. M. Chan et al., "Red and processed meat and colorectal cancer incidence: Meta-analysis of prospective studies," PLoS One, vol. 6, no. 6, 2011, doi: 10.1371/journal.pone.0020456.

[28] H. U. Krämer, B. Schöttker, E. Raum, and H. Brenner, "Type 2 diabetes mellitus and colorectal cancer: Meta-analysis on sexspecific differences," Eur. J. Cancer, vol. 48, no. 9, pp. 12691282, 2012, doi: 10.1016/j.ejca.2011.07.010.

[29] G. Nakatsu et al., "Gut mucosal microbiome across stages of colorectal carcinogenesis," Nat. Commun., vol. 6, pp. 30-32, 2015, doi: 10.1038/ncomms9727.
[30] A. D. Kostic et al., "Fusobacterium nucleatum Potentiates Intestinal Tumorigenesis and Modulates the Tumor-Immune Microenvironment," Cell Host Microbe, vol. 14, no. 2, pp. $207-$ 215, 2013, doi: 10.1016/j.chom.2013.07.007.

[31] B. Levin et al., "Screening and Surveillance for the Early Detection of Colorectal Cancer and Adenomatous Polyps, 2008: A Joint Guideline from the American Cancer Society, the US Multi-Society Task Force on Colorectal Cancer, and the American College of Radiology," CA. Cancer J. Clin., vol. 58, no. 3, pp. 130-160, 2008, doi: 10.3322/ca.2007.0018.

[32] Pan American Health Organization, "Tamizaje de Cáncer Colorrectal en las Américas Situación y Retos," 2014. https://www.paho.org/hq/dmdocuments/2016/ColorrectalCancer--Screening-Landscape-report-Espanol.pdf (accessed Jan. 14, 2021).

[33] C. Hassan et al., "Meta-analysis: Adherence to colorectal cancer screening and the detection rate for advanced neoplasia, according to the type of screening test," Aliment. Pharmacol. Ther., vol. 36, no. 10, pp. 929-940, 2012, doi: 10.1111/apt.12071.

[34] D. J. Robertson et al., "Recommendations on Fecal Immunochemical Testing to Screen for Colorectal Neoplasia: A Consensus Statement by the US Multi-Society Task Force on Colorectal Cancer," Gastroenterology, vol. 152, no. 5, pp. 1217-1237.e3, 2017, doi: 10.1053/j.gastro.2016.08.053.

[35] J. Tinmouth, I. Lansdorp-Vogelaar, and J. E. Allison, "Faecal immunochemical tests versus guaiac faecal occult blood tests: What clinicians and colorectal cancer screening programme organisers need to know," Gut, vol. 64, no. 8, pp. 1327-1337, 2015, doi: 10.1136/gutjnl-2014-308074.

[36] T. F. Imperiale et al., "Multitarget Stool DNA Testing for Colorectal-Cancer Screening," N. Engl. J. Med., vol. 370, no. 14, pp. 1287-1297, 2014, doi: 10.1056/nejmoa1311194.

[37] J. B. Kisiel and P. J. Limburg, "Colorectal Cancer Screening With the Multitarget Stool DNA Test," Am. J. Gastroenterol., vol. 115, no. 11, pp. 1737-1740, 2020, doi: 10.14309/ajg.0000000000000968.

[38] F. Stracci, M. Zorzi, and G. Grazzini, "Colorectal Cancer Screening: Tests, Strategies, and Perspectives," Front. Public Heal., vol. 2, no. October, pp. 1-9, 2014, doi: 10.3389/fpubh.2014.00210.

[39] J. E. Shin, "Quality Indicators for Colonoscopy and the Risk of Interval Cancer (New England Journal of Medicine 2010;362:1795-1803)," Intest. Res., vol. 8, no. 1, p. 91, 2010, doi: 10.5217/ir.2010.8.1.91.

[40] A. I. Neugut and S. Pita, "Role of sigmoidoscopy in screening for colorectal cancer: A critical review," Gastroenterology, vol. 95, no. 2, pp. 492-499, 1988, doi: 10.1016/00165085(88)90511-2.

[41] W. H. Organization and I. P. on C. Safety, "Biomarkers in risk assessment: validity and validation." World Health Organization, Geneva PP - Geneva, 2001, [Online]. Available: https://apps.who.int/iris/handle/10665/42363.

[42] K. Strimbu and J. A. Tavel, "What are biomarkers?," Curr. Opin. HIV AIDS, vol. 5, no. 6, pp. 463-466, 2010, doi: 10.1097/COH.0b013e32833ed177.

[43] D. Sidransky, "Emerging molecular markers of cancer," Nat. Rev. Cancer, vol. 2, no. 3, pp. 210-219, 2002, doi: $10.1038 /$ nrc755.

[44] NIH, "Cáncer - Instituto Nacional del Cáncer," 2021. https://www.cancer.gov/espanol (accessed Jan. 15, 2021).

[45] World Health Organization WHO, "INTERNATIONAL PROGRAMME ON CHEMICAL SAFETY," 1993.

[46] M. Gonzalez-Pons and M. Cruz-Correa, "Colorectal Cancer Biomarkers: Where Are We Now?," Biomed Res. Int., vol. 2015, no. Table 1, 2015, doi: 10.1155/2015/149014.

[47] M. Kalia, "Personalized oncology: Recent advances and future challenges," Metabolism., vol. 62, no. SUPPL.1, pp. S11-S14, 2013, doi: 10.1016/j.metabol.2012.08.016.

[48] B. T. Pan and R. M. Johnstone, "Fate of the transferrin receptor during maturation of sheep reticulocytes in vitro: Selective externalization of the receptor," Cell, vol. 33, no. 3, pp. 967978, 1983, doi: 10.1016/0092-8674(83)90040-5.

[49] C. Frühbeis, D. Fröhlich, and E. M. Krämer-Albers, "Emerging 
roles of exosomes in neuron-glia communication," Front. Physiol., vol. 3 APR, no. April, pp. 1-7, 2012, doi: 10.3389/fphys.2012.00119.

[50] C. Frühbeis et al., "Neurotransmitter-Triggered Transfer of Exosomes Mediates Oligodendrocyte-Neuron Communication," PLoS Biol., vol. 11, no. 7, 2013, doi: 10.1371/journal.pbio.1001604.

[51] V. Luga et al., "Exosomes mediate stromal mobilization of autocrine Wnt-PCP signaling in breast cancer cell migration," Cell, vol. 151, no. 7, pp. 1542-1556, 2012, doi: 10.1016/j.cell.2012.11.024

[52] V. R. Minciacchi, M. R. Freeman, and D. Di Vizio, "Extracellular Vesicles in Cancer: Exosomes, Microvesicles and the Emerging Role of Large Oncosomes," Semin. Cell Dev. Biol., vol. 40, pp. 41-51, 2015, doi: 10.1016/j.semcdb.2015.02.010

[53] K. Denzer, M. J. Kleijmeer, H. F. G. Heijnen, W. Stoorvogel, and H. J. Geuze, "Exosome: From internal vesicle of the multivesicular body to intercellular signaling device," J. Cell Sci., vol. 113, no. 19, pp. 3365-3374, 2000.

[54] C. Théry, M. Ostrowski, and E. Segura, "Membrane vesicles as conveyors of immune responses," Nat. Rev. Immunol., vol. 9, no. 8, pp. 581-593, 2009, doi: 10.1038/nri2567.

[55] B. Roucourt, S. Meeussen, J. Bao, P. Zimmermann, and G. David, "Heparanase activates the syndecan-syntenin-ALIX exosome pathway," Cell Res., vol. 25, no. 4, pp. 412-428, 2015, doi: 10.1038/cr.2015.29.

[56] B. György et al., "Membrane vesicles, current state-of-the-art: Emerging role of extracellular vesicles," Cell. Mol. Life Sci., vol. 68, no. 16, pp. 2667-2688, 2011, doi: 10.1007/s00018-0110689-3.

[57] M. F. Baietti et al., "Syndecan-syntenin-ALIX regulates the biogenesis of exosomes," Nat. Cell Biol., vol. 14, no. 7, pp. 677-685, 2012, doi: 10.1038/ncb2502.

[58] M. Colombo et al., "Analysis of ESCRT functions in exosome biogenesis, composition and secretion highlights the heterogeneity of extracellular vesicles," J. Cell Sci., vol. 126, no. 24 , pp. 5553-5565, 2013, doi: 10.1242/jcs. 128868 .

[59] E. R. Abels and X. O. Breakefield, "Introduction to Extracellular Vesicles: Biogenesis, RNA Cargo Selection, Content, Release, and Uptake," Cell. Mol. Neurobiol., vol. 36, no. 3, pp. 301-312, 2016, doi: 10.1007/s10571-016-0366-z.

[60] A. O. Fadaka, A. Pretorius, and A. Klein, "Biomarkers for Stratification in Colorectal Cancer: MicroRNAs," Cancer Control, vol. 26, no. 1, pp. 1-11, 2019, doi: $10.1177 / 1073274819862784$.

[61] J. T. Henry and B. Johnson, "Current and evolving biomarkers for precision oncology in the management of metastatic colorectal cancer," Chinese Clin. Oncol., vol. 8, no. 5, pp. 1-14, 2019, doi: 10.21037/cco.2019.08.08.

[62] D. Plano, V. Alcolea, C. Sanmartín, and A. K. Sharma, "Methods of selecting combination therapy for colorectal cancer patients: a patent evaluation of US20160025730A1," Expert Opin. Ther. Pat., vol. 27, no. 5, pp. 527-538, 2017, doi: 10.1080/13543776.2017.1315103.

[63] W. De Roock et al., "Effects of KRAS, BRAF, NRAS, and PIK3CA mutations on the efficacy of cetuximab plus chemotherapy in chemotherapy-refractory metastatic colorectal cancer: A retrospective consortium analysis," Lancet Oncol., vol. 11 , no. 8, pp. 753-762, 2010, doi: 10.1016/S14702045(10)70130-3.

[64] H. Davies et al., "Mutations of the BRAF gene in human cancer," Nature, vol. 417, no. 6892, pp. 949-954, 2002, doi: 10.1038/nature00766.

[65] Y. Xiao et al., "Exosomes as potential sources of biomarkers in colorectal cancer," Cancer Letters, vol. 476. pp. 13-22, 2020, doi: 10.1016/j.canlet.2020.01.033.

[66] H. Ogata-Kawata et al., "Circulating exosomal microRNAs as biomarkers of colon cancer," PLoS One, vol. 9, no. 4, 2014, doi: 10.1371/journal.pone.0092921.

[67] A. G. Lalkhen and A. McCluskey, "Clinical tests: Sensitivity and specificity," Contin. Educ. Anaesthesia, Crit. Care Pain, vol. 8, no. 6, pp. 221-223, 2008, doi: 10.1093/bjaceaccp/mkn041.
[68] L. Ayers, R. Pink, D. R. F. Carter, and R. Nieuwland, "Clinical requirements for extracellular vesicle assays," J. Extracell. Vesicles, vol. 8, no. 1, 2019, doi: 10.1080/20013078.2019.1593755.

[69] N. Karimi et al., "Serum overexpression of miR-301a and miR23a in patients with colorectal cancer," J. Chinese Med. Assoc., vol. 82, no. 3, pp. 215-220, 2019, doi: 10.1097/JCMA.0000000000000031.

[70] X. Liu et al., "DNA-methylation-mediated silencing of miR486-5p promotes colorectal cancer proliferation and migration through activation of PLAGL2/IGF2/ $\beta$-catenin signal pathways," Cell Death Dis., vol. 9, no. 10, 2018, doi: 10.1038/s41419-018-1105-9.

[71] S. Yan et al., "Exosomal miR-6803-5p as potential diagnostic and prognostic marker in colorectal cancer," J. Cell. Biochem., vol. 119, no. 5, pp. 4113-4119, 2018, doi: $10.1002 /$ jcb.26609.

[72] L. Min et al., "Evaluation of circulating small extracellular vesicles derived miRNAs as biomarkers of early colon cancer: a comparison with plasma total miRNAs," J. Extracell. Vesicles, vol. 8, no. 1, 2019, doi: 10.1080/20013078.2019.1643670.

[73] M. S. Ostenfeld et al., "miRNA profiling of circulating EpCAM+ extracellular vesicles: promising biomarkers of colorectal cancer," J. Extracell. Vesicles, vol. 5, no. 1, 2016, doi: 10.3402/jev.v5.31488.

[74] B. J. Desmond, E. R. Dennett, and K. M. Danielson, "Circulating extracellular vesicle microRNA as diagnostic biomarkers in early colorectal cancer-A review," Cancers (Basel)., vol. 12, no. 1, 2020, doi: 10.3390/cancers12010052.

[75] A. Q. Gomes, S. Nolasco, and H. Soares, "Non-coding RNAs: Multi-tasking molecules in the cell," Int. J. Mol. Sci., vol. 14, no. 8, pp. 16010-16039, 2013, doi: 10.3390/ijms140816010.

[76] T. Liu et al., "Exosomal long noncoding RNA CRNDE-h as a novel serum-based biomarker for diagnosis and prognosis of colorectal cancer," Oncotarget, vol. 7, no. 51, pp. 8555185563, 2016, doi: 10.18632/oncotarget.13465.

[77] L. Dong et al., "Circulating long RNAs in serum extracellular vesicles: Their characterization and potential application as biomarkers for diagnosis of colorectal cancer," Cancer Epidemiol. Biomarkers Prev., vol. 25, no. 7, pp. 1158-1166, 2016, doi: 10.1158/1055-9965.EPI-16-0006.

[78] C. Barbagallo et al., "LncRNA UCA1, Upregulated in CRC Biopsies and Downregulated in Serum Exosomes, Controls mRNA Expression by RNA-RNA Interactions," Mol. Ther. Nucleic Acids, vol. 12, no. September, pp. 229-241, 2018, doi: 10.1016/j.omtn.2018.05.009.

[79] Y. Chen et al., "Protein content and functional characteristics of serum-purified exosomes from patients with colorectal cancer revealed by quantitative proteomics," Int. J. Cancer, vol. 140, no. 4, pp. 900-913, 2017, doi: 10.1002/ijc.30496.

[80] C. Campanella et al., "Heat shock protein 60 levels in tissue and circulating exosomes in human large bowel cancer before and after ablative surgery," Cancer, vol. 121, no. 18, pp. 32303239, 2015, doi: 10.1002/cncr.29499.

[81] J. Li et al., "GPC1 exosome and its regulatory miRNAs are specific markers for the detection and target therapy of colorectal cancer," J. Cell. Mol. Med., vol. 21, no. 5, pp. 838847, 2017, doi: 10.1111/jcmm.12941.

[82] B. Sun et al., "Circulating exosomal CPNE3 as a diagnostic and prognostic biomarker for colorectal cancer," J. Cell. Physiol., vol. 234, no. 2, pp. 1416-1425, 2019, doi: 10.1002/jcp.26936.

[83] M. E. Zhong et al., "Serum extracellular vesicles contain SPARC and LRG1 as biomarkers of colon cancer and differ by tumour primary location," EBioMedicine, vol. 50, pp. 211-223, 2019, doi: 10.1016/j.ebiom.2019.11.003. 